Old Dominion University

ODU Digital Commons

Physics Faculty Publications

Physics

$1-1999$

\title{
Role of Spontaneous Emission in Ultracold Two- Color Optical Collisions
}

\author{
C. I. Sukenik \\ Old Dominion University, csukenik@odu.edu
}

T. Walker

Follow this and additional works at: https://digitalcommons.odu.edu/physics_fac_pubs

Part of the Atomic, Molecular and Optical Physics Commons

\section{Repository Citation}

Sukenik, C. I. and Walker, T., "Role of Spontaneous Emission in Ultracold Two-Color Optical Collisions" (1999). Physics Faculty Publications. 14.

https://digitalcommons.odu.edu/physics_fac_pubs/14

\section{Original Publication Citation}

Sukenik, C.I., \& Walker, T. (1999). Role of spontaneous emission in ultracold two-color optical collisions. Physical Review A, 59(1), 889-892. doi: 10.1103/PhysRevA.59.889 


\title{
Role of spontaneous emission in ultracold two-color optical collisions
}

\author{
C. I. Sukenik* and T. Walker \\ Department of Physics, University of Wisconsin-Madison, Madison, Wisconsin 53706
}

(Received 27 July 1998)

\begin{abstract}
We have observed violet photon emission resulting from energy-pooling collisions between ultracold $\mathrm{Rb}$ atoms illuminated by two colors of near-resonant infrared laser light. We have used this emission as a probe of doubly excited state ultracold collision dynamics. By varying the detuning of the lasers, we have clearly identified the effect of spontaneous emission on the collision process. [S1050-2947(99)08701-6]
\end{abstract}

PACS number(s): 32.80.Pj, 34.50.Gb, 33.80.-b

Ultracold collisions of atoms differ from "room", temperature collisions in a number of important ways [1]. Long de Broglie wavelengths imply that quantum effects often dominate the ultracold collision dynamics, whereas classical trajectories work well at room temperature. Furthermore, the durations of ultracold collisions approach microseconds, as compared to picoseconds at room temperature, or, more significantly, as compared to excited-state spontaneous emission lifetimes of tens of nanoseconds. Thus the coupling of the colliding atoms to the vacuum modes of the electromagnetic field plays an essential role in determining excited-state collision dynamics. Any realistic treatment of excited-state ultracold collision processes must therefore include these dissipative effects which are very difficult to account for in conventional quantum scattering theory. Accordingly, substantial theoretical effort has been expended to develop new methods for treating excited-state ultracold collisions [2]. On the experimental side, careful studies of the effects of spontaneous emission have encountered substantial difficulties for the alkali-metal atoms due to the complexity of the excited-state hyperfine structure [3-5] while lack of hyperfine complexity has allowed a fairly good understanding of single-photon collisions for metastable $\mathrm{Xe}[6,7]$ and $\mathrm{He}$ [8]. In this paper we present a study of the effects of spontaneous emission on two-color collisions.

For single-color ultracold collisions that result in trap loss, a laser field excites two colliding atoms to a strongly attractive excited-state potential curve $(5 S+5 P$ in the case of $\mathrm{Rb}$ ). The atoms are accelerated towards each other. When the atoms emit a photon due to spontaneous emission, they leave the trap provided the energy gained on the excited-state potential exceeds (twice) the depth of the trap. Since the survival probability roughly obeys $\exp [-t / \tau]$, where $t$ is the time on the excited-state potential and $\tau$ is the natural lifetime of the excited state, the trap-loss process strongly favors excited-state potential curves that have long lifetimes $[9,10]$. Two-color ultracold collisions are quite different in this respect. A two-color collision (Fig. 1) begins by absorption of a photon to a singly excited state, in much the same way as a single-photon collision, but the acceleration is terminated not by spontaneous emission but by absorption from a second laser field to a doubly excited state (DES, $5 P+5 P$ ).

\footnotetext{
*Present address: Dept. of Physics, Old Dominion University,
} Norfolk, VA 23529.
Once on the weakly attractive DES, the atoms proceed toward each other, and spontaneous emission can terminate the collision. If the atoms reach small interatomic separations before spontaneous emission occurs, inelastic energy transfer produces an ion ( $\mathrm{Na}$ [11]) or photon ( $\mathrm{Rb}$ [12]) that is observed. From this argument, it is easy to see how the effects of spontaneous emission differ for two-color collisions as compared to single-photon collisions. First, the time spent on the singly excited potential curve is very small, so that spontaneous emission during the acceleration process can be ignored. A corollary to this is that the two-color process does not favor the singly excited potential curves with long lifetimes, favoring rather those curves with the strongest couplings to the ground state. Second, spontaneous emission is
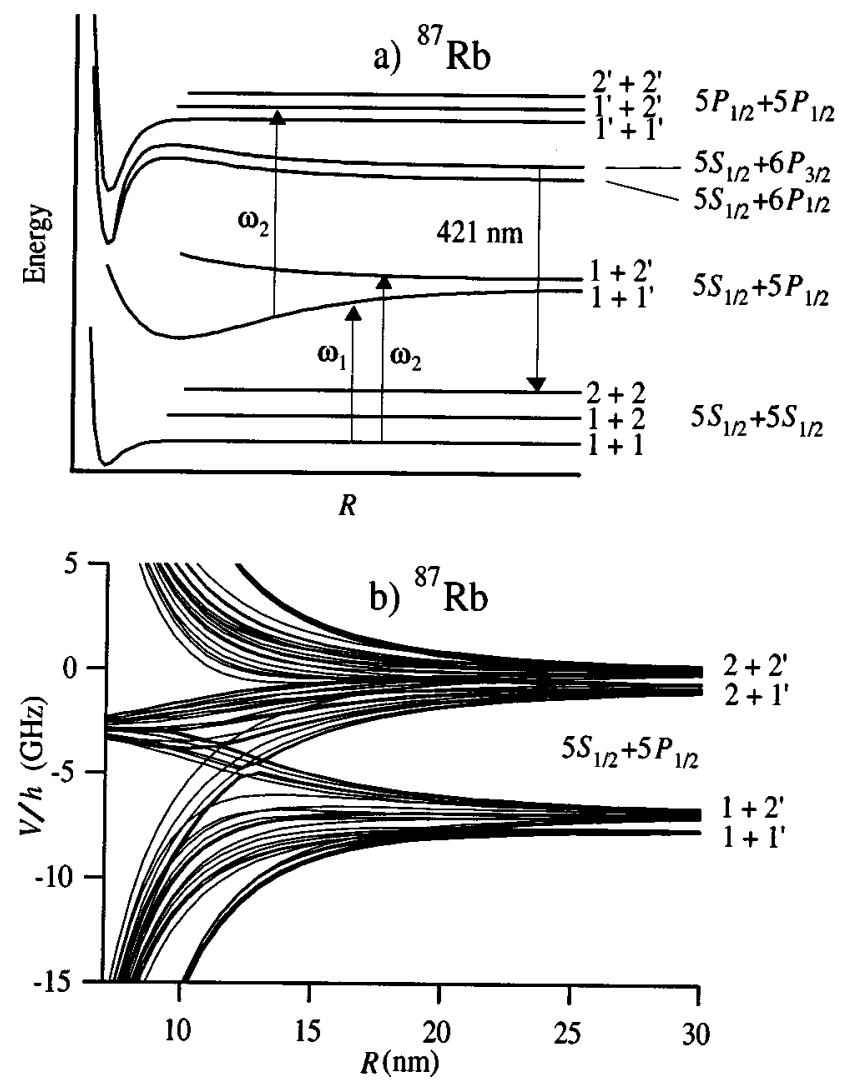

FIG. 1. (a) Schematic representation of ${ }^{87} \mathrm{Rb}$ energy levels involved in the two-photon optical collision; (b) calculated ${ }^{87} \mathrm{Rb}$ potential curves showing hyperfine structure of the singly excited $5 S_{1 / 2}+5 P_{1 / 2}$ manifold. 
important once the atoms are on the DES. Again, since the DES lifetimes are all comparable to atomic lifetimes, no particular DES states are singled out. Third, since the amount of acceleration in the two-color process is determined by the frequency difference between the two photons that are absorbed, it is easy to vary the spontaneous emission probability by varying the frequency difference of the two lasers.

We recently reported a two-color collision experiment in $\mathrm{Rb}$ in which we studied many other unique aspects of twocolor collisions, including extremely low saturation intensities due to optical shielding and population depletion, and observation of Condon oscillations in the frequency dependence of the energy pooling rate [12]. In this Brief Report we concentrate on those aspects of the two-color process that are sensitive to spontaneous emission, and show how spontaneous emission dramatically suppresses collision rates for some collision channels and competes with optical shielding in others.

We confine either ${ }^{87} \mathrm{Rb}$ or ${ }^{85} \mathrm{Rb}$ atoms in a standard magneto-optical trap (MOT), the apparatus being similar to that described previously $[13,14]$. We apply two laser frequencies $\omega_{1}$ and $\omega_{2}$ (with intensities $I_{1}$ and $I_{2}$ ) which can cause excitations to the $5 P+5 P$ level. An atom pair in this state that reaches small interatomic separation can undergo energy pooling to $5 S+6 P$ states and the outgoing $6 P$ atom subsequently decays with emission of a violet photon at 421 $\mathrm{nm}$. We measure the rate at which these violet photons are produced as we vary $\omega_{2}$. Since the frequency range explored here $\left(0.1 \mathrm{~cm}^{-1}\right)$ is much less than the energy transfer $(\sim 1400$ $\mathrm{cm}^{-1}$ ), we expect that any frequency dependence in the rate at which violet photons are produced comes from lightinduced dynamics at large interatomic separation, not from changes in the energy-pooling probability at small interatomic separations. We use the violet photon production rate, therefore, as a probe of the DES collision dynamics.

We give important details of our experiment here; a comprehensive account of the experiment and the semiclassical model we developed to interpret our results will be published elsewhere [15]. In total, five frequencies are derived from three external-cavity diode lasers. Two frequencies are used to produce the MOT, one frequency provides optical pumping as explained below, and the two frequencies used to study the collisions are $\omega_{1}$ and $\omega_{2} . \omega_{1}$ is detuned $\Delta_{1}=$ $-90 \mathrm{MHz}$ from the $5 S_{1 / 2}(F=1) \rightarrow 5 P_{1 / 2}\left(F^{\prime}=1\right)$ atomic transition (for ${ }^{87} \mathrm{Rb}$ ), or the $(F=2) \rightarrow\left(F^{\prime}=2\right)$ transition (for ${ }^{85} \mathrm{Rb}$ ), and populates attractive $5 S_{1 / 2}+5 P_{1 / 2}$ curves. Excitation by $\omega_{1}$ typically takes place at an interatomic separation between 10 and $40 \mathrm{~nm}$. The other laser frequency $\omega_{2}$ is detuned $\Delta_{2}=0-2 \mathrm{GHz}$ above the $5 S_{1 / 2}(F=1)$ $\rightarrow 5 P_{1 / 2}\left(F^{\prime}=2\right)$ atomic transition (for ${ }^{87} \mathrm{Rb}$ ), or the $(F$ $=2) \rightarrow\left(F^{\prime}=3\right.$ ) transition (for ${ }^{85} \mathrm{Rb}$ ) (Fig. 1). All lasers can be switched on and off using acousto-optic modulators. Acousto-optic modulator frequency shifts are included in the above frequencies. The MOT lasers are switched off when $\omega_{1}$ and $\omega_{2}$ are present to reduce the number of collisional pathways. For a typical sequence the trap laser is turned on for $80 \mu$ s, followed by $2 \mu$ s of optical pumping to put nearly all the atoms into the lowest ground-state level, and, finally, $15 \mu$ s of $\omega_{1}$ and $\omega_{2}$. We detect violet photons with a photomultiplier tube or charge-coupled-device camera. Given small count rates $\left(<10 \mathrm{~s}^{-1}\right)$, we carefully filtered out all

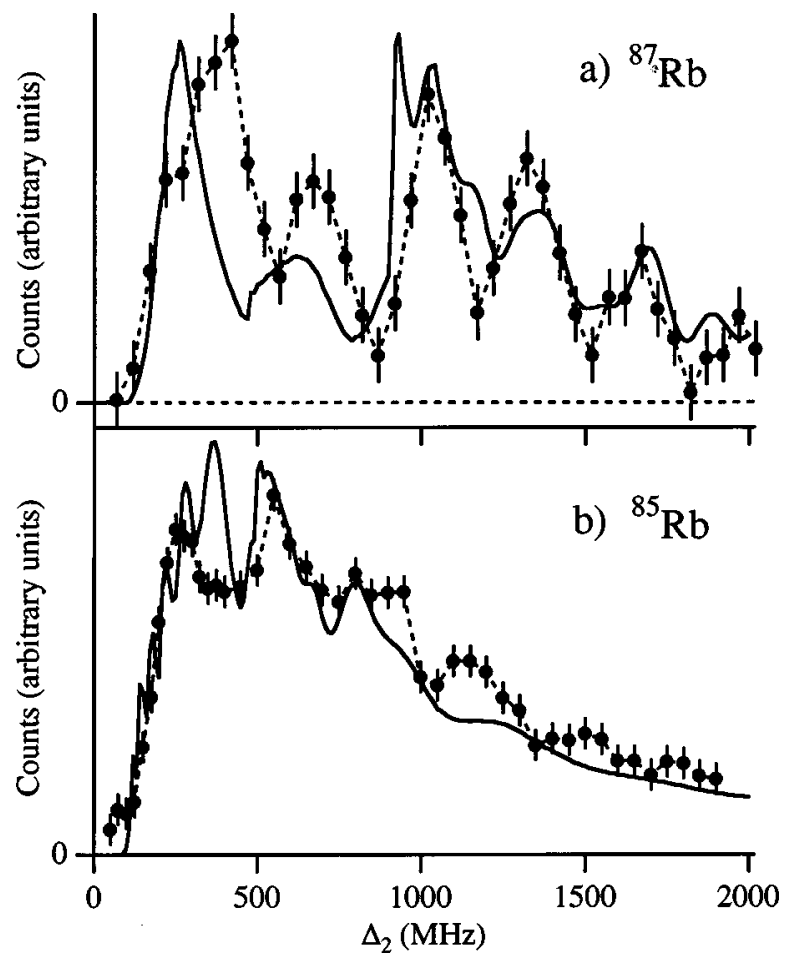

FIG. 2. Violet photon production rate as a function of $\Delta_{2}$ for (a) ${ }^{87} \mathrm{Rb}$ and (b) ${ }^{85} \mathrm{Rb}$. The solid line shows results from a calculation described in the text.

scattered near-IR light using a series of filters which yielded an extinction ratio of greater than $10^{12}$ for near-IR photons while only attenuating the violet photons by $\sim 60 \%$ [16].

We now describe briefly the overall results of this experiment to put the effects of spontaneous emission into context. As shown in Fig. 2, there is a strong dependence of the violet photon production rate on $\omega_{2}$, including an unexpected appearance of deep modulations in the ${ }^{87} \mathrm{Rb}$ spectrum. As it turns out, these modulations contain a wealth of information about the collision process. We find the modulations themselves arise primarily because of Franck-Condon (FC) overlap factors for the second excitation and the onset of new collisional channels. The amplitude of the modulationsparticularly close to the channel thresholds-also contains information about two other processes: optical shielding and spontaneous emission.

Optical shielding has been studied in a number of previous experiments [17]. Here it occurs when the atom pair is excited from the ground state to a repulsive curve of the singly excited $5 S_{1 / 2}+5 P_{1 / 2}$ state. When this happens, incoming flux is rerouted at long range and the atom pair does not reach a sufficiently close separation to be excited to attractive curves by $\omega_{1}$. Shielding produces a quite low saturation intensity [12] $\left(\sim 30 \mathrm{~mW} / \mathrm{cm}^{2}\right)$ for small $\Delta_{2}$.

As discussed in Ref. [12], two channels of the $5 P+5 P$ manifold are observed in this experiment. The onset of these new channels is evidenced by the rising slopes of the first and third peaks in Fig. 2(a). For example, the first peak, whose onset is at $90 \mathrm{MHz}$, results from excitation to the $1^{\prime}$ $+2^{\prime}$ DES [18] and the third peak originates predominantly from excitation to the $2^{\prime}+2^{\prime}$ state. Note that the frequency difference in the onset of the peaks corresponds to the 812.5 MHz hyperfine splitting of the excited state. Other "peaks" 
in the data are modulations introduced by the FC overlap factors for excitation by $\omega_{2}$.

In order to understand the interplay between the various collision processes, we developed a semiclassical model including the full hyperfine structure of the potential curves. Near the channel thresholds spontaneous emission on the DES becomes important because the atoms receive a small impulse on the SES curves. The velocity scale below which spontaneous emission is important is given by $v=R / \tau$ $\sim 100 \mathrm{~cm} / \mathrm{s}$, where $R \sim 30-40 \mathrm{~nm}$ is the position of the $\omega_{2}$ Condon point. This translates into a detuning from threshold of roughly $50 \mathrm{MHz}$. Near a threshold, then, spontaneous emission manifests itself as a reduction in the flux of atoms reaching small $R$, with a corresponding drop in the violet photon emission rate.

At first sight, the separation of shielding and spontaneous emission effects seems difficult. However, the hyperfine splitting in the DES allows us to isolate them. The point is that shielding only effects one of the two final-state channels, as can be understood from Fig. 1. For the $1^{\prime}+2^{\prime}$ channel, the threshold is at small values of both $\Delta_{1}$ and $\Delta_{2}$, i.e., both colors are nearly resonant with the atomic resonances and in particular the Condon point for $\Delta_{2}$ to excite the atoms to repulsive SES curves is at large interatomic separation. On the other hand, for the $2^{\prime}+2^{\prime}$ channel the two-color resonance is at $\Delta_{2}=902 \mathrm{MHz}$, at which detuning the Condon point for excitation to repulsive SES curves has moved to small interatomic separation. Thus both shielding and spontaneous emission affect violet photon production near the $1^{\prime}+2^{\prime}$ threshold, but only spontaneous emission affects it for the $2^{\prime}+2^{\prime}$ case.

We illustrate the effects of spontaneous emission and shielding by comparing our data to a detailed model of the collisions, which is described elsewhere [15]. We begin by determining all the long-range molecular potentials, including hyperfine structure, of the states involved [19]. Next, we calculate the excitation probability to each SES hyperfine curve using the two-level Landau-Zener (LZ) curve crossing model for excitation [2], explicitly including the FC wavefunction overlap factor [20]. Semiclassical WKB wave functions are used for the SES and an $s$-wave ground-state wave function with published scattering lengths [21]. The Rabi frequency used in the LZ formula is calculated using a magnetic-sublevel averaged matrix element. For all excitations, we average over the intensity of the standing waves of $\omega_{1}$ and $\omega_{2}$, which are both retroreflected after passing through the MOT. Optical shielding by $\omega_{2}$ and flux depletion of the ground state [12] arise naturally in the model as flux is removed from the ground state at each Condon point. Once excited by $\omega_{1}$, we allow the atoms to approach each other classically. Along their trajectory they may become resonant with $\omega_{2}$ before decaying and be excited to one of the three hyperfine levels of the DES. The excitation probability to the DES is again calculated according to the LZ model. The wave functions for the three DES hyperfine levels are taken to be sinusoidal with an adjustable phase treated as a free parameter. Lastly, we calculate the survival probability in the DES to reach small separation $(<1 \mathrm{~nm})$, where we assume a violet photon is produced with a probability independent of

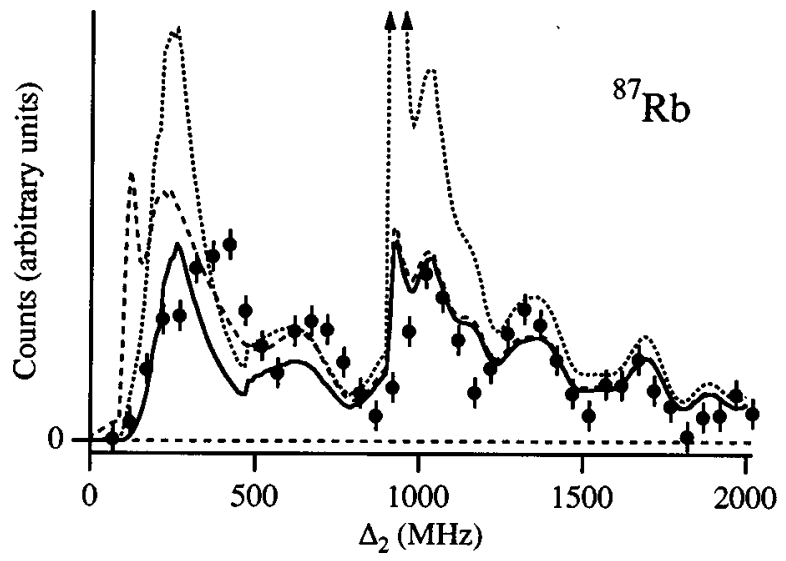

FIG. 3. Data (points) and calculation (solid line) as in Fig. 2(a) with additional results from the calculation neglecting only spontaneous emission (dotted line) and neglecting only optical shielding (dashed line).

$\omega_{2}$. We have used a single initial relative velocity of 20 $\mathrm{cm} / \mathrm{s}$. Including a distribution of velocities did not appreciably change the results.

This semiclassical model, while much more comprehensive than any others used to date in excited-state ultracold collisions, is clearly not complete. While $s$ waves are certainly very important, higher-order partial waves ( $p$ and perhaps $d$ waves as well) should also contribute to the signal. By averaging over magnetic sublevels, we have neglected possible effects due to local optical pumping. Since the excitation probability at a given Condon point is small, using a magnetically averaged oscillator strength is likely a good approximation. For similar reasons, our treatment of the Condon points as independent is probably fairly accurate. These approximations are necessitated by both tractability and an incomplete knowledge of all relevant wave functions. Nonetheless, our model allows us to interpret our results in some detail.

We now focus on the first and third "peaks" of Fig. 2(a). Recall that these peaks correspond to the onset of new collision channels as $\omega_{2}$ becomes resonant with the two upper hyperfine levels of the DES. The first peak, where $\Delta_{2}$ is small, is sensitive to both shielding and spontaneous emission. By contrast, the onset of the new collisional channel as evidenced by the third peak at around $900 \mathrm{MHz}$ is modified by spontaneous emission, but not optical shielding. In Fig. 3 we have replotted Fig. 2(a), this time including additional results from our calculation. In the figure, the data showing the violet photon production rate as a function of $\Delta_{2}$ are displayed with three predictions of the model calculation. The solid line is the full calculation, as shown in Fig. 2(a). The dotted line shows results of the calculation when the effect of spontaneous emission has been eliminated. And finally, the dashed line is a plot of the model results if we simply ignore optical shielding. Notice that both optical shielding and spontaneous emission play an important part in suppressing violet photon production at low detunings, but it is spontaneous emission alone which suppresses the violet photon production around $900 \mathrm{MHz}$. Also, even though there is a nonvanishing oscillator strength to the lowest of the three DES hyperfine levels below an $\omega_{2}$ detuning of 90 
$\mathrm{MHz}$, violet photon production there is very strongly suppressed by optical shielding. The reduction in violet photon production is made all the more dramatic by the fact that at detunings corresponding to the onset of new channels, where the relative velocities are low, the excitation probability to the DES is greatly enhanced by the slow speeds (the denominator in the Landau-Zener formula is large).

In conclusion, we have unambiguously demonstrated the critical role of spontaneous emission, and its relationship to optical shielding, in doubly excited state ultracold collisions. The impact of spontaneous emission is most pronounced when the blue-detuned laser is near a two-color resonance but away from a one-photon atomic resonance. Understanding of this collision process was facilitated by comparison with a hyperfine structure-inclusive model.

We acknowledge support from the NSF and the Packard Foundation.
[1] See T. Walker and P. Feng, Adv. At., Mol., Opt. Phys. 34, 125 (1994); J. Weiner, ibid. 35, 45 (1995).

[2] K.-A. Suominen, Y. B. Band, I. Tuvi, K. Burnett, and P. S. Julienne, Phys. Rev. A 57, 3724 (1998).

[3] D. Hoffmann, P. Feng, R. Williamson, and T. Walker, Phys. Rev. Lett. 69, 753 (1992); D. Hoffmann, P. Feng, and T. Walker, J. Opt. Soc. Am. B 11, 712 (1994).

[4] M. G. Peters, D. Hoffmann, J. Tobiason, and T. Walker, Phys. Rev. A 50, R906 (1994).

[5] S. D. Gensemer and P. L. Gould, Phys. Rev. Lett. 80, 936 (1998).

[6] K. Suominen, K. Burnett, P. Julienne, M. Walhout, U. Sterr, C. Orzel, M. Hoogerland, and S. Rolston, Phys. Rev. A 53, 1678 (1996).

[7] C. Orzel, S. Bergeson, S. Kulin, and S. Rolston, Phys. Rev. Lett. 80, 5093 (1998).

[8] H. Mastwijk, J. Thomsen, P. van der Straten, and A. Niehaus, Phys. Rev. Lett. 80, 5516 (1998).

[9] P. Julienne and J. Vigue, Phys. Rev. A 44, 4464 (1991).

[10] P. Lett, K. S. Gensemer, K. Tan, A. Kumarakrishnan, C. Wal- lace, and P. Gould, J. Phys. B 28, 65 (1995).

[11] V. Bagnato, L. Marcassa, C. Tsao, Y. Wang, and J. Wiener, Phys. Rev. Lett. 70, 3225 (1993).

[12] C. I. Sukenik, D. Hoffmann, S. Bali, and T. Walker, Phys. Rev. Lett. 81, 782 (1998).

[13] D. Hoffmann et al., J. Opt. Soc. Am. B 11, 712 (1994).

[14] P. Feng and T. Walker, Am. J. Phys. 63, 905 (1995).

[15] C. I. Sukenik, D. Hoffmann, and T. Walker (unpublished).

[16] Schott BG39 and Melles Griot 03 FIV 026.

[17] R. Napolitano et al., Phys. Rev. A 55, 1191 (1997), and references therein.

[18] As we measure $\Delta_{2}$ from the $5 S_{1 / 2}(F=1) \rightarrow 5 P_{1 / 2}\left(F^{\prime}=2\right)$ atomic transition (for ${ }^{87} \mathrm{Rb}$ ), the $1^{\prime}+1^{\prime}$ peak would occur at negative $\Delta_{2}$.

[19] T. Walker and D. Pritchard, Laser Phys. 4, 1085 (1994).

[20] N. F. Mott and H. S. W. Massey, The Theory of Atomic Collisions, 3rd ed. (Oxford University Press, London, 1965).

[21] N. R. Newbury et al., Phys. Rev. A 51, R2680 (1995); J. R. Gardner et al., Phys. Rev. Lett. 74, 3764 (1995). 